\title{
Review Article \\ New Oral Anticoagulants in the Treatment of Pulmonary Embolism: Efficacy, Bleeding Risk, and Monitoring
}

\author{
Kelly M. Rudd ${ }^{1}$ and Elizabeth (Lisa) M. Phillips ${ }^{2}$ \\ ${ }^{1}$ Department of Pharmaceutical Care Services, Bassett Medical Center, Cooperstown, NY 13326, USA \\ ${ }^{2}$ Wegmans School of Pharmacy, St. John Fisher College, Rochester, NY 14618, USA \\ Correspondence should be addressed to Kelly M. Rudd; kelly.rudd@bassett.org
}

Received 22 June 2012; Accepted 31 January 2013

Academic Editor: C. Arnold Spek

Copyright ( 2013 K. M. Rudd and E. M. Phillips. This is an open access article distributed under the Creative Commons Attribution License, which permits unrestricted use, distribution, and reproduction in any medium, provided the original work is properly cited.

\begin{abstract}
Anticoagulation therapy is mandatory in patients with pulmonary embolism to prevent significant morbidity and mortality. The mainstay of therapy has been vitamin-K antagonist therapy bridged with parenteral anticoagulants. The recent approval of new oral anticoagulants (NOACs: apixaban, dabigatran, and rivaroxaban) has generated significant interest in their role in managing venous thromboembolism, especially pulmonary embolism due to their improved pharmacokinetic and pharmacodynamic profiles, predictable anticoagulant response, and lack of required efficacy monitoring. This paper addresses the available literature, on-going clinical trials, highlights critical points, and discusses potential advantages and disadvantages of the new oral anticoagulants in patients with pulmonary embolism.
\end{abstract}

\section{Introduction}

Pulmonary embolism (PE) is a common, potentially fatal disease with an annual incidence of approximately 70 cases per 100000 people [1,2]. Patients generally require hospitalization and recurrence is common [3]. In addition to significant morbidity and mortality, treatment costs associated with thrombosis and the arising postthrombotic syndromes are staggering, with costs exceeding 500 million dollars annually in the United States $[1,4,5]$.

The mainstay of treatment for the past fifty years has been warfarin therapy, overlapped with a parenteral anticoagulant until the vitamin $\mathrm{K}$ antagonist (VKA) is fully therapeutic [2, 5]. While highly effective at reducing morbidity and mortality associated with PE, VKA therapy poses challenges, including variability in drug response, patient compliance, and drugdrug, drug-disease, and drug-diet interactions [6-9]. Great interest has been generated regarding the recently marketed new oral anticoagulants (NOACs) and their potential role as alternative anticoagulant therapies.

\section{Risk Assessment in Pulmonary Embolism}

Pulmonary embolism is a serious and potentially fatal complication of a venous thrombotic event (VTE). Despite clinical trials yielding similar estimates for safety and efficacy in overall treatment when comparing deep vein thrombosis (DVT) and PE, patients with PE incur additional risks not seen in patients with DVT alone. The impact of PE on mortality is striking, with significant increases in the risk of death at both 30 and 90 days after event [10]. In addition to increasing mortality, PE also has significant impact on morbidity. Cardiorespiratory impairment, including pulmonary hypertension, can result in chronic and irreversible health concerns. Additionally, recurrent episodes of either VTE or PE are approximately $40 \%$ higher in patients with history of a PE, propagating the dangers of VTE and PE into the future $[5,10]$. These factors culminate in an essential need for safe and effective anticoagulant treatments for pulmonary embolism.

Three new oral anticoagulants (apixaban, dabigatran, and rivaroxaban) are the newest to enter the anticoagulant 
armamentarium. The available data on each agent in the treatment of $\mathrm{PE}$, their respective mechanisms of action, pharmacokinetic profiles, and potential places in therapy are discussed.

\section{Relevant Pharmacokinetic and Pharmacodynamic Clinical Pearls}

Each of the NOACs has unique pharmacokinetic and pharmacodynamic properties that clinicians must consider when selecting oral anticoagulant therapy. The comparative pharmacokinetic profiles for the NOACs in respect to warfarin are provided in Table 1 . The newer agents have a more rapid onset and shorter duration of activity than warfarin. This suggests that standard management of starting a parenteral anticoagulant simultaneous to an oral VKA (bridging) therapy upon initiation or interruption of therapy may become unnecessary in some patients. An important caveat to this change is that some Phase II and III data indicate a potential need for higher doses for some of the NOACs during the first few weeks of treating an acute thrombotic event, requiring close attention to dosing recommendations and adjustments $[11,12]$. Clinical trials with dabigatran, however, have utilized a study design requiring a lead-in treatment period with a parenteral anticoagulant prior to the initiation of study medication thereby making a decision about the utility of dabigatran as primary treatment for VTE an unanswered clinical question $[13,14]$. The reader is directed to Section 4 of this paper for more details on available clinical trials.

An equally important point is the dosing schedules of the NOACs range from once to twice daily, despite relatively short half-lives. This makes compliance, appropriate counseling and monitoring imperative components to successful therapy. Although the NOACs do not have the dietary restrictions of warfarin, an important caveat for rivaroxaban is that higher doses (i.e., >10 mg/day) need to be given with food to enhance bioavailability. Additionally, the rivaroxaban package insert recommends avoiding administration via feeding tube due to concerns of decreased bioavailability. While there are no dietary restrictions with dabigatran, the dosage form cannot be crushed or chewed as bioavailability increases well above therapeutic levels. Dabigatran must also be stored in the original container to prevent capsule degradation, with the medication expiring 4 months after opening of the original container. Dabigatran therefore cannot be placed in a pill box or organizer because of its hygroscopic tendencies.

The NOACs have fewer drug interactions than warfarin; however vigilance with patient's medication profiles and concomitant medications is necessary as the NOACs are subject to drug-drug interactions via the cytochrome p450 and p-glycoprotein systems (Table 2). Note that the suggested dosing alterations provided in Table 2 for dabigatran and apixaban are those noted in the package inserts currently approved under the indications of nonvalvular atrial fibrillation (AF). In many cases, the treatment doses utilized for PE are higher than those approved for other indications, making caution necessary when applying available dose adjustment recommendations to patients with VTE. If these agents receive FDA approval for the treatment of $\mathrm{PE}$, updates to the package inserts to address drug interactions and recommendations for adjustment to the newly approved doses will be imperative. As rivaroxaban is now approved for the treatment of PE as well as nonvalvular AFIB and DVT prevention, the table highlights the interactions consistent with these dosing schedules.

Lastly, the varying degree of renal elimination and altered response in patients with evidence of renal dysfunction adds to the clinical complexity for the appropriate patient selection. Dabigatran, rivaroxaban, and apixaban have FDA approved dosing adjustments for patients with nonvalvular $\mathrm{AF}$ and who have compromised renal function. For dabigatran and rivaroxaban, these renal dosing recommendations, while FDA-approved, are based upon pharmacokinetic studies and have not been studied clinically. When apixaban was studied, the clinical trials took into account the patients level of renal function and so the dose adjustment in the package insert is based on validated data from the clinical trial. Therefore, the FDA approved dose for apixiban is to reduce the dose in patients with two of the following characteristics: age $\geq 80$ years, body weight $<60 \mathrm{~kg}$ or serum creatinine $\geq 1.5 \mathrm{mg} / \mathrm{dL}$ (Table 2 ). Additional considerations are necessary in patients with both renal dysfunction and concurrent drug-drug interactions, as the pharmacokinetics become even more uncertain. Consideration for continued use and referral to a specialized anticoagulation clinic or service offers the prescriber with a comprehensive assessment of the appropriateness of patient selection for the NOACs.

The predictable pharmacokinetic profile of the newer NOACs results in less variability in drug response thereby negating the need for individualized therapeutic monitoring as required with VKA. Although the newer agents can alter coagulation assays (Table 1), there is an inconsistent relationship between drug effect and assay response to allow clinical utility of laboratory monitoring. Although there is a clinically appealing feature of these new agents, this poses a therapeutic challenge when bleeding occurs or urgent reversal is required, as traditional coagulation assays do not provide quantitative evidence for the intensity of anticoagulant effect. However, these assays can be used to qualitatively assess the presence of drug and thereby guide course of action in the event of emergency. Conversely, given the relative insensitivity of traditional assays (such as the INR and aPTT) to the NOACs, normalization of the assay may not always indicate lack of drug or complete reversal of the pharmacologic effect $[15,16]$.

The lack of a reversal agent for the NOACs remains one of the most important clinical concerns with these new agents. As the NOACs pharmacologically do not induce clotting factor deficiencies as a mechanism of action, the utility of replacement products such as fresh frozen plasma (FFP) is limited. Since the NOACs represent inhibitors of the coagulation cascade, reversal will remain a challenge until an antidote is developed and tested clinically.

Currently, only summary recommendations regarding the management of bleeding or "reversal" of the NOACs can be made. Although there are published studies, many are ex vivo or animal studies that cannot reliably predict an in-vivo 
TABLE 1: Comparison of the pharmacokinetic and pharmacodynamic features of oral anticoagulant therapies [15, 18, 21, 23-31].

\begin{tabular}{llll}
\hline Characteristic & Warfarin & Apixaban & Dabigatran \\
\hline Mechanism of action & VKORC1 enzyme inhibitor & Direct ${ }^{\wedge}$ Factor Xa inhibitor & $\begin{array}{l}\text { Direct } \\
\text { (Thrombin) inhibitor }\end{array}$ \\
\hline Prodrug & No & No & Yes \\
\hline & $\begin{array}{l}\text { VTE prevention } \\
\text { VTE treatment }\end{array}$ & $\begin{array}{l}\text { VTE prevention } \\
\text { (EMA, HC) }\end{array}$ & VTE prevention \\
Approved indications & $\begin{array}{l}\text { Atrial fibrillation } \\
\text { Valvular heart disease } \\
\text { (all approved in EMA, HC, }\end{array}$ & $\begin{array}{l}\text { Nonvalvular AF } \\
\text { (EMA, HC, FDA) }\end{array}$ & $\begin{array}{l}\text { Nonvalvular AF } \\
\text { (EMA, HC, FDA) }\end{array}$ \\
& FDA) & & \\
\hline
\end{tabular}

Agent can cause major or fatal bleeding; Regular INR monitoring is necessary;

FDA black box warnings drugs, dietary changes, and other factors affect INR levels (FDA)
Discontinuation in patients without adequate continuous anticoagulation None to date increases the risk of stroke (FDA)

Rivaroxaban Direct $^{\wedge}$ Factor Xa inhibitor

No

VTE prevention

(EMA, HC, FDA)

Nonvalvular AF

(EMA, HC, FDA)

VTE treatment (EMA, HC, FDA)

Discontinuing places patients at an increased risk of thrombotic events; Risk of spinal/epidural hematoma during neuraxial anesthesia/spinal puncture (FDA)

$V T E$ prevention and nonvalvular AF: Fixed, once daily With dose reduction

Fixed, twice daily With dose reduction in two of the following patients: (per FDA)

Dosing Variable, patient specific
(1) Age $\geq 80$ years

(2) Weight $\leq 60 \mathrm{~kg}$

(3) Serum creatinine $\geq 1.5 \mathrm{mg} / \mathrm{dL}$
Fixed, twice daily With dose reduction in patients with renal dysfunction (per FDA) in patients with renal dysfunction (per FDA)

VTE treatment: Fixed, twice daily $x$ 3 weeks then fixed, once daily Not to be used if $\mathrm{ClCr}$ $<30 \mathrm{~mL} / \mathrm{min}$

\begin{tabular}{lllll}
\hline$T_{\max }(\mathrm{h})$ & 4 & $1-3$ & $1-3$ & $2-4$ \\
\hline Half-life (h) & $20-60$ & 12 & $12-17$ & $5-9$ \\
\hline
\end{tabular}

$10 \mathrm{mg}$ : 80-100\%

(regardless of food) 20 mg: 66\% (without Bioavailability (F)

$100 \%$

$50 \%$

$6 \%$

( $\sim 75 \%$ if capsule opened)

food).
Food increases absorption ${ }^{\#}$

$36 \%$ unchanged drug*

$33 \%$ as inactive metabolites Mostly via secretion not glomerular filtration

$>90 \% \quad 15 \%$

(S-enantiomer is a substrate (primary substrate for

CYP metabolism ${ }^{+}$ for 2C9 and 2C19 while CYP3A4; minor No $30 \%$ $\mathrm{R}$-enantiomer is a substrate contributions by others, for $1 \mathrm{~A} 1,1 \mathrm{~A} 2$, and $3 \mathrm{~A} 4) \quad$ without active metabolites)

\begin{tabular}{lllll}
\hline P-glycoprotein $(\mathrm{P}-\mathrm{gp})^{+}$ & No & Yes & Yes & Yes
\end{tabular}

VTE prevention dose: does not specify AFIB: Take with evening meal VTE treatment: Take with food 
TABLe 1: Continued.

\begin{tabular}{|c|c|c|c|c|}
\hline Characteristic & Warfarin & Apixaban & Dabigatran & Rivaroxaban \\
\hline \multicolumn{5}{|l|}{$\begin{array}{l}\text { Influence on routine } \\
\text { coagulation assay }\end{array}$} \\
\hline Protime (PT) & $\uparrow$ & $\uparrow$ & $\uparrow$ & $\uparrow$ \\
\hline $\mathrm{aPTT}$ & $\mathrm{No} / \uparrow$ & $\uparrow$ & $\uparrow$ & $\uparrow$ \\
\hline Thrombin time (TT) & No & No & $\uparrow \uparrow$ & No \\
\hline $\begin{array}{l}\text { Coagulation assay } \\
\text { used to monitor efficacy }\end{array}$ & INR (Protime) & None & None & None \\
\hline $\begin{array}{l}\text { Clinically validated } \\
\text { Reversal agent }\end{array}$ & $\begin{array}{l}\text { Vitamin K, FFP, PCC, } \\
\text { Factor VIIa, aPCC }\end{array}$ & None & None & None \\
\hline
\end{tabular}

EMA: European Medicines Agency; HC: Health Canada; FDA: U.S. Food and Drug Administration

AF: Atrial Fibrillation; CYP: cytochrome P450; VKORC1: C1 subunit of the vitamin K epoxide reductase enzyme; FFP: Fresh Frozen Plasma; PCC: Prothrombin Complex Concentrate.

${ }^{\wedge}$ Does not bind to antithrombin.

${ }^{\#}$ Rivaroxaban $15 \mathrm{mg}$ tablet, if available, should be taken with food.

${ }^{*}$ Dose adjustment for level of renal dysfunction required.

${ }^{+}$Potential source for drug-drug interactions-review full package insert for details.

response and therefore recommendations remain inconclusive. In vitro publications in humans are sparse and primarily limited to case reports [17]. For reversal of dabigatran, the use of activated charcoal and dialysis in cases of life threatening bleeding or overdose can be considered [18]. Activated Factor VIIa (Novoseven) is a seemingly attractive option though it has not been studied in humans. In animal models, bleeding complications were not reliably and consistently reversed [19], and use in the nonhemophiliac population has been associated with an increased risk of thrombosis [20].

A nonactivated 4-factor PCC product (Cofact) failed to show reversal in coagulation parameters induced by dabigatran in a randomized, placebo-controlled, crossover study in healthy subjects. In this study, bleeding was not an accessible outcome [15]. van Ryn and colleagues performed an in vitro study using nonactivated 4-factor PCCs (Beriplex and Octaplex), an activated PCC product (FEIBA), and an activated Factor VII (Novoseven) and found a reduced bleeding time after administration, despite failure to reverse coagulation parameters such as aPTT and thrombin time [16]. These animal model studies suggest a potential lack of correlation between changes in coagulation parameters and bleeding for dabigatran, and further study is needed to confirm this hypothesis [15].

In regard to rivaroxaban, dialysis is not a viable option due to high protein binding (92-95\%) [21], and no data on activated charcoal could be found. Animal studies using activated Factor VII (Novoseven) show only modest effect on reducing bleeding, and there are no human clinical data to support routine use [22]. Additionally, the concern of increased risk of thrombosis remains with activated Factor VII use [19]. A randomized, placebo-controlled, crossover study in healthy subjects suggested that a nonactivated 4-factor PCC product (Cofact) used in healthy adults could reverse rivaroxaban effect on the PT; however it is unknown whether this translates into decreasing bleeding [15].

No data or recommendations currently exist for reversal of apixaban therapy. To complicate translation of this research into clinical practice, nonactivated 4-factor PCCs are unavailable in the United States, and the only 4-factor PCC available is the activated FEIBA product. Institutions are therefore left with the development of institution-specific policies for reversal based upon the foundation of supportive care and local measures to stop bleeding, the availability of the local medical expertise, an understanding of available literature and reversal agents, and cost.

\section{Anticoagulant Therapy for Treatment of Pulmonary Embolism}

4.1. Apixaban. Limited data exist to determine the safety and efficacy of apixaban for the treatment of PE. The AMPLIFY (NCT00643201) trial is a Phase III, randomized, doubleblind, double-dummy, noninferiority trial ongoing to assess apixaban in the initial treatment of VTE [33]. AMPLIFY is currently enrolling patients in a 6-month study, comparing dose-adjusted warfarin (INR goal 2.0-3.0) with parenteral anticoagulant bridge to apixaban $10 \mathrm{mg}$ twice daily for 7 days followed by apixaban $5 \mathrm{mg}$ tablets twice daily. The study is expected to enroll 4816 in 471 study locations and is expected to close in March 2013 with a primary outcome of a composite time to recurrent VTE or death [33]. No safety or efficacy data are presently available from the AMPLIFY trial.

AMPLIFY-EXT is a randomized, double-blind study evaluating the safety and efficacy of apixaban for the extended treatment of DVT and PE over a 12-month period following the initial 6-12 months of anticoagulation therapy [34]. Subjects were randomized to apixaban $2.5 \mathrm{mg}$ or $5 \mathrm{mg}$ twice daily or placebo and were eligible if they were 18 years of age or older, had objective confirmed proximal DVT of the leg(s) or PE, had finished 6-12 months of standard anticoagulant therapy, or had completed treatment with apixaban or enoxaparin and warfarin as part of the AMPLIFY trial, had no symptomatic recurrent of VTE during prior anticoagulant therapy, and had clinical equipoise to continue or cease anticoagulation therapy. Patients were excluded if they 
TABLE 2: Selected P-glycoprotein and Cytochrome P450 3A4 drug interactions with NOAC based on current FDA-approved indications $[18,21,31,32]$.

\begin{tabular}{|c|c|c|c|c|}
\hline Medications $^{+}$ & Mechanism of Interaction & $\begin{array}{l}\text { Dabigatran }{ }^{\#} \\
\text { (Pradaxa) }\end{array}$ & $\begin{array}{l}\text { Rivaroxaban }{ }^{\wedge} \\
(\text { Xarelto) }\end{array}$ & $\begin{array}{l}\text { Apixaban } \\
\text { (Eliquis) }\end{array}$ \\
\hline Rifampin & P-glycoprotein inducer & Avoid combination & Avoid & Avoid \\
\hline $\begin{array}{l}\text { Carbamazepine, phenytoin, } \\
\text { St. John's wort }\end{array}$ & $\begin{array}{l}\text { P-glycoprotein inducers } \\
\text { and } \\
\text { Strong 3A4 inducers }\end{array}$ & Avoid & Avoid & Avoid \\
\hline
\end{tabular}

\begin{tabular}{|c|c|c|c|c|}
\hline Dronedarone & P-glycoprotein inhibitors & $\begin{array}{l}\text { For } \mathrm{CrCl} 30-50 \mathrm{~mL} / \mathrm{min} \text { : } \\
\text { Dose reduction suggested } \\
\text { in dosing for Atrial } \\
\text { Fibrillation } \\
\text { For } \mathrm{CrCl}<30 \mathrm{~mL} / \mathrm{min} \text { : } \\
\text { Aviod }\end{array}$ & $\begin{array}{l}\text { Not addressed in } \\
\text { Package Insert }\end{array}$ & Not addressed \\
\hline $\begin{array}{l}\text { Systemic ketoconazole } \\
\text { Itraconazole }\end{array}$ & $\begin{array}{l}\text { P-glycoprotein inhibitors } \\
\text { and } \\
\text { Strong 3A4 inhibitors }\end{array}$ & $\begin{array}{l}\text { For } \mathrm{CrCl} 30-50 \mathrm{~mL} / \mathrm{min} \text { : } \\
\text { Dose reduction suggested } \\
\text { in dosing for Atrial } \\
\text { Fibrillation }\end{array}$ & Avoid & $\begin{array}{l}\text { Reduce dose in } 1 / 2 \text { for } \\
\text { those starting on the full } \\
\text { dose of } 5 \mathrm{mg} \mathrm{BID}^{\%}\end{array}$ \\
\hline $\begin{array}{l}\text { lopinavir/ritonavir, } \\
\text { ritonavir, } \\
\text { indinavir/ritonavir, } \\
\text { conivaptan }\end{array}$ & $\begin{array}{l}\text { P-glycoprotein inhibitors } \\
\text { and } \\
\text { Strong 3A4 inhibitor }\end{array}$ & $\begin{array}{l}\text { Not addressed in Package } \\
\text { Insert }\end{array}$ & Avoid & $\begin{array}{l}\text { Reduce dose in } 1 / 2 \text { for } \\
\text { those starting on the full } \\
\text { dose of } 5 \mathrm{mg} \mathrm{BID} \%\end{array}$ \\
\hline $\begin{array}{l}\text { Verapamil, amiodarone, } \\
\text { quinidine, }\end{array}$ & P-glycoprotein inhibitors & $\begin{array}{l}\text { For } \mathrm{CrCl} 30-50 \mathrm{~mL} / \mathrm{min} \text { : } \\
\text { No dose adjustment } \\
\text { needed, monitor clinical } \\
\text { course } \\
\text { For } \mathrm{CrCl}<30 \mathrm{~mL} / \mathrm{min} \text { : } \\
\text { Aviod }\end{array}$ & $\begin{array}{l}\text { Not addressed in } \\
\text { Package Insert }\end{array}$ & $\begin{array}{l}\text { Reduce dose in } 1 / 2 \text { for } \\
\text { those starting on the full } \\
\text { dose of } 5 \mathrm{mg} \mathrm{BID} \%\end{array}$ \\
\hline Clarithromycin & $\begin{array}{l}\text { P-glycoprotein inducers } \\
\text { and strong 3A4 inducers }\end{array}$ & $\begin{array}{l}\text { For } \mathrm{CrCl}<30 \mathrm{~mL} / \mathrm{min} \\
\text { Avoid } \\
\text { For } \mathrm{CrCl} 30-50 \mathrm{~mL} / \mathrm{min} \text { : } \\
\text { No dose adjustment } \\
\text { needed, monitor clinical } \\
\text { course }\end{array}$ & $\begin{array}{l}\text { Unclear: refer to } \\
\text { package insert. }\end{array}$ & $\begin{array}{l}\text { Reduce dose in } 1 / 2 \text { for } \\
\text { those starting on the full } \\
\text { dose of } 5 \mathrm{mg} \mathrm{BID} \%\end{array}$ \\
\hline
\end{tabular}

CrCl: creatinine clearance as determined by Crockcoft-Gault equation.

\#Dabigatran etexilate (prodrug) uses the p-glycoprotein transport system. It is not a substrate, inducer, or inhibitor of the cytochrome p450 system.

${ }^{\wedge}$ Rivaroxaban is a substrate for cytochrome p450 as well as p-glycoprotein.

${ }^{+}$Listed medications are representatives of potent $3 \mathrm{~A} 4$ inhibitors and may not be comprehensive.

${ }^{\%}$ Those patients who need to start at $2.5 \mathrm{mg}$ BID should avoid this combination.

had contraindication to continued anticoagulant therapy, had dual antiplatelet therapy, were on aspirin doses greater than $165 \mathrm{mg}$ daily, had elevated liver function tests, or has depressed hematologic markers.

The majority of patients on apixaban in AMPLIFY-EXT weighed greater than $60 \mathrm{~kg}$ (92.6\%). Serum creatinines (for dose adjustment, per the package insert) were not provided; though the majority of patients on apixaban (99.8\%) had a calculated creatinine clearance greater than $30 \mathrm{~mL} / \mathrm{min}$, 91.8\% had a calculated creatinine clearance greater than $50 \mathrm{~mL} / \mathrm{min}$. Of the subjects on apixaban, $98.3 \%$ completed 612 months of prior anticoagulation therapy, as opposed to less than 6 months and more than 12 months, though no further information was provided as to the exact discontinuation date of the initial anticoagulant therapy. Patients lost to follow were classified as having the primary outcome event and negative for reaching the primary safety endpoint. Study endpoints are presented in Table 3.
Based upon information provided by the authors of the source article, apixaban $5 \mathrm{mg}$ twice daily lowered the rate of recurrent, nonfatal pulmonary embolism [23], when therapy was extended one year beyond the initial anticoagulation period when compared to placebo. Until the results from AMPLIFY are available, it remains unknown if apixaban is safe and effective in the primary treatment of VTE with pulmonary embolism [33].

4.2. Dabigatran Etexilate Mesylate. Dabigatran, an oral direct thrombin inhibitor, has been FDA approved for nonvalvular atrial fibrillation and is also approved in Europe and Canada for VTE prevention. Currently, dabigatran is not approved for the treatment of VTE in Canada, Europe, or the United States of America, and applications to the respective regulatory bodies (EMA, HC, and FDA) have not been submitted. Presently four Phase III clinical trials, RE-COVER, RE-COVER-II, REMEDY (NCT00329238), and RE-SONATE (NCT00558259), 
TABLE 3: Outcomes of apixaban in AMPLIFY-EXT [34].

\begin{tabular}{|c|c|c|c|}
\hline Outcome & $\begin{array}{l}\text { Apixaban } 2.5 \mathrm{mg} \\
(n=840 ; 13 \mathrm{LTF})\end{array}$ & $\begin{array}{l}\text { Apixaban } 5 \mathrm{mg} \\
(n=813 ; 20 \mathrm{LTF})\end{array}$ & $\begin{array}{l}\text { Placebo } \\
(n=829 ; 19 \mathrm{LTF})\end{array}$ \\
\hline \multirow{3}{*}{$\begin{array}{l}\text { Primary efficacy endpoint: composite of symptomatic recurrent vte or } \\
\text { death from any cause }\end{array}$} & $32(3.8 \%)$ & $34(4.2 \%)$ & \multirow{3}{*}{$96(11.6 \%)$} \\
\hline & RR versus placebo & RR versus placebo & \\
\hline & $0.33(0.22-0.48)$ & $0.36(0.25-0.53)$ & \\
\hline \multirow{3}{*}{$\begin{array}{l}\text { Secondary efficacy endpoint: symptomatic recurrent VTE or death } \\
\text { related to VTE }\end{array}$} & $14(1.7 \%)$ & $14(1.7 \%)$ & \multirow{3}{*}{$73(8.8 \%)$} \\
\hline & RR versus placebo & RR versus placebo & \\
\hline & $0.19(0.11-0.33)$ & $0.20(0.11-0.34)$ & \\
\hline \multirow{3}{*}{$\begin{array}{l}\text { Additional endpoint added after trial initiation: composite of } \\
\text { symptomatic recurrent VTE, death related to VTE, myocardial } \\
\text { infarction, stroke, or death related to cardiovascular cause }\end{array}$} & $18(2.1 \%)$ & $19(2.3 \%)$ & \multirow{3}{*}{$83(10 \%)$} \\
\hline & RR versus placebo & RR versus placebo & \\
\hline & $0.21(0.13-0.35)$ & $0.23(0.14-0.38)$ & \\
\hline \multirow{3}{*}{ Primary safety endpoint: major bleeding ${ }^{\wedge}$} & $2(0.2 \%)$ & $1(0.1 \%)$ & \multirow{3}{*}{$4(0.5 \%)$} \\
\hline & RR versus placebo & RR versus placebo & \\
\hline & $0.49(0.09-2.64)$ & $0.25(0.03-2.24)$ & \\
\hline \multirow{3}{*}{ 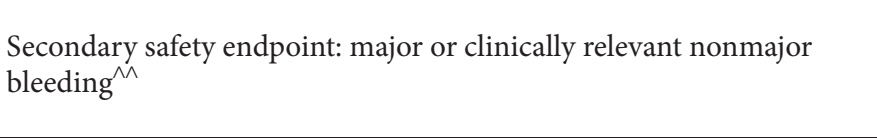 } & $27(3.2 \%)$ & $35(4.3 \%)$ & \multirow{3}{*}{$22(2.7 \%)$} \\
\hline & RR versus placebo & RR versus placebo & \\
\hline & $1.2(0.69-2.10)$ & $1.62(0.96-2.73)$ & \\
\hline \multirow{3}{*}{ Recurrent fatal PE or death where PE could not be excluded } & $2(0.2 \%)$ & $3(0.4 \%)$ & \multirow{3}{*}{$7(0.8 \%)$} \\
\hline & RR versus placebo & RR versus placebo & \\
\hline & $0.28(0.06-1.35)$ & $0.43(0.11-1.68)$ & \\
\hline \multirow{3}{*}{ Recurrent nonFatal PE } & $8(1 \%)$ & $4(0.5 \%)$ & \multirow{3}{*}{$15(1.8 \%)$} \\
\hline & RR versus placebo & RR versus placebo & \\
\hline & $0.53(0.22-1.23)$ & $0.27(0.09-0.82)$ & \\
\hline Study conclusion & \multicolumn{3}{|c|}{$\begin{array}{l}\text { Extension with } 12 \text { months of apixaban } 2.5 \mathrm{mg} \text { or } 5 \mathrm{mg} \text { twice daily } \\
\text { therapy reduced the risk of recurrent VTE and recurrent nonfatal } \\
\text { PE, without increasing the risk of major bleeding. }\end{array}$} \\
\hline
\end{tabular}

NR: not reported, LTF: lost to follow (classified as having the primary efficacy endpoint).

${ }^{\wedge}$ Major bleeding as defined in AMPLIFY-EXT: clinical overt bleeding, with associated fall in hemoglobin of at least $2 \mathrm{~g}$ per deciliter, the need for transfusion of 2 or more units of red blood cells, involving a critical site or was fatal.

${ }^{\wedge}$ Nonmajor bleeding as defined in AMPLIFY-EXT: bleeding that did not meet criteria for major bleeding but AHT was associated with the need for medical intervention, unscheduled contact with a physician, interruption or discontinuation of the study drug, or discomfort or impairment of activities of daily living. In addition, any bleeding compromising hemodynamics, leading to hospitalization, development of subcutaneous hematoma larger than $25 \mathrm{~cm}^{2}$ or $100 \mathrm{~cm}^{2}$ if traumatic cause, intramuscular hematoma documented by ultrasonography, epistaxis of more than 5 minutes duration, or was repetitive, spontaneous gingival bleeding, spontaneous macroscopic hematuria lasting great than 24 hours, macroscopic gastrointestinal hemorrhage, and hemoptysis not occurring with PE.

have been completed to assess the efficacy of dabigatran in the treatment of VTE, with or without PE, though only RECOVER has published full results $[13,14,35,36]$.

RE-COVER is a randomized, double-blind, double-dummy, noninferiority trial conducted in 228 centers world-wide, enrolling 2564 patients [14]. Patients were eligible if they were 18 years of age or older and had objective confirmed proximal DVT of the leg(s) or PE. Patients were excluded if they had VTE symptoms for more than 14 days, experiencing hemodynamic instability requiring thrombolytic therapy, had recent cardiovascular event, or were at high risk for bleeding.

The design of RECOVER was unique in that all patients received a parenteral anticoagulant (intravenous unfractionated heparin or subcutaneous low molecular weight heparin) for the first 6 days, while warfarin dosing was being adjusted to achieve a target INR of $2-3$ by sham INR. After that time, patients continued their blinded randomized assignment of either Dabigatran (150 mg twice daily) or warfarin (doseadjusted to a target INR of 2.0-3.0). Because parenteral anticoagulation was used during the first week, larger initial dabigatran doses, as seen in other NOACs studies, were not utilized as part of this protocol.

RE-COVER II was of similar design, enrolling 2568 patients and was conducted to confirm the previous results and gather data sufficient for subgroup analyses [35]. Study endpoints are presented in Table 4.

All RE-COVER study patients on dabigatran had a creatinine clearance above $50 \mathrm{~mL} / \mathrm{min}$ (mean $105.8 \pm 40.7$ ) with a median weight of $84 \mathrm{~kg}$ (range: $38-175$ ). The proportion of cancer patients, those with the highest risk of development and morbidity associated with PE, were small in the dabigatran and warfarin groups, $5.0 \%$ and $4.5 \%$, respectively [14].

Subgroup analysis did not indicate differences in the primary endpoint in relation to gender, age, or race or those presenting with initial symptomatic PE or active cancer at 
TABle 4: Outcomes of dabigatran in VTE studies [14, 35].

\begin{tabular}{|c|c|c|}
\hline Outcome & $\begin{array}{l}\text { RE-COVER } \\
\text { (dabigatran versus warfarin) }\end{array}$ & $\begin{array}{l}\text { RE-COVER II } \\
\text { (dabigatran versus warfarin) }\end{array}$ \\
\hline $\begin{array}{l}\text { Primary efficacy endpoint: } \\
\text { recurrent symptomatic VTE or } \\
\text { VTE-Related Death }\end{array}$ & $2.4 \%$ versus $2.1 \%$, HR 1.10 (95\% CI $0.65-1.84)$ & $2.4 \%$ versus $2.2 \%$, HR 1.08 (95\% CI $0.64-1.8$ ) \\
\hline $\begin{array}{l}\text { Secondary efficacy endpoint: } \\
\text { recurrent, nonfatal PE }\end{array}$ & $1.0 \%$ versus $0.6 \%$, HR $1.85(07.4-4.64)$ & NR \\
\hline $\begin{array}{l}\text { Total days overlap between } \\
\text { warfarin/dabigatran and parenteral } \\
\text { anticoagulant }\end{array}$ & $5-10$ days $($ mean $=10$ days $)$ & 5-11 days (mean NR) \\
\hline Major Bleeding $\wedge$ & $1.6 \%$ versus $1.9 \%$, HR 0.82 (95\% CI $0.45-1.48)$ & $1.1 \%$ versus $1.7 \%$, HR 0.69 (95\% CI $0.36-1.32)$ \\
\hline $\begin{array}{l}\text { Major or clinically relevant } \\
\text { non-major bleeding }\end{array}$ & $5.6 \%$ versus $8.8 \%$, HR 0.63 (95\% CI $0.47-0.84)$ & NR \\
\hline Any bleeding & $16.1 \%$ versus $21.9 \%$, HR 0.71 (95\% CI $0.59-0.85)$ & $15.6 \%$ versus $22.1 \%$, HR 0.67 (95\% CI $0.56-0.81)$ \\
\hline Death & $1.6 \%$ versus $1.7 \%$, HR $0.95(0.53-1.79)$ & $1.9 \%$ in both groups \\
\hline Warfarin TTR & $60 \%$ & NR \\
\hline Study conclusion & $\begin{array}{l}\text { Dabigatran is noninferior to warfarin } \\
\text { Dabigatran is not superior to warfarin }\end{array}$ & $\begin{array}{l}\text { Dabigatran is noninferior to warfarin } \\
\text { Dabigatran is not superior to warfarin }\end{array}$ \\
\hline
\end{tabular}

NR: not reported, TTR: time in therapeutic range.

${ }^{\wedge}$ Major bleeding as defined in RE-COVER: Clinical overt bleeding, with associated fall in hemoglobin of at least $20 \mathrm{~g}$ per liter, the need for transfusion of 2 or more units of red blood cells, involving a critical site or was fatal.

${ }^{\wedge}$ Minor bleeding as defined in RE-COVER: spontaneous skin hematoma of at least $25 \mathrm{~cm}^{2}$, spontaneous nose bleed of more than 5 minutes in duration, macroscopic hematuria lasting more than 24 hours, spontaneous rectal bleeding, gingival bleeding of greater than 5 minutes, bleeding leading to hospitalization and/or requiring surgical treatment, bleeding leading to transfusion of less than 2 units of red blood cells, or any other clinically relevant bleeding per the investigator.

baseline. Likewise, the rate of recurrent VTE in patients with previous VTE was not statistically different in the two treatment groups $(P=0.09)$, though trended to favor warfarin therapy [14].

Comparison data were not available in RE-COVER II as this was presented in abstract form only. Study authors note that a full publication is pending.

In RE-COVER, dabigatran $150 \mathrm{mg}$ twice daily was noninferior, but not superior to dose-adjusted warfarin managed to a time in therapeutic range (TTR) of 60 percent. The rate of recurrent $\mathrm{PE}$ was higher in the dabigatran group (1\% versus $0.6 \%$, HR $1.85,95 \%$ CI $0.74-4.64$ ), though not statistically different. The dabigatran $150 \mathrm{mg}$ twice daily dose was also utilized in the RE-LY atrial fibrillation study. Noting that the 18113 patients were not randomized to correct for VTE risk factors, nor were they reported, the rates of PE development in the dabigatran and warfarin groups were $0.15 \%$ per year and $0.09 \%$ per year respectively (Relative Risk 1.61, 95\% CI $0.76-3.42, P=0.21$, warfarin mean TTR 64\%) [14].

The RE-MEDY (NCT00329238) and RE-SONATE (NCT00558259) trials have both been completed, with data collection terminating in October 2010 and February 2011, respectively. RE-MEDY is a randomized, double-blind, multicenter, parallel-group, active controlled study to compare the efficacy and safety of dabigatran $150 \mathrm{mg}$ twice daily to dose-adjusted warfarin (INR target 2.0-3.0) for the secondary prevention of VTE. A total of 2867 patients in 275 study locations were followed for a total of 36 months to the primary outcome of composite recurrent VTE or VTE death at 18 and 36 months [13].
RE-SONATE is a randomized, double-blind, multicenter, parallel-group, prevention study to compare dabigatran $150 \mathrm{mg}$ twice daily versus matching placebo in the longterm prevention of recurrent, symptomatic VTE in patients with history of DVT or PE who completed 6-18 months of treatment with VKA therapy. Total study enrollment is 1353 patients in 147 study locations. The primary study endpoint is time to centrally confirmed VTE over the 6-month study period [36].

Bleeding remains a main safety concern with dabigatran and the NOACs. In the setting of acute VTE, warfarin showed slightly higher rates of nonmajor bleeding, though no difference in major bleeding outcomes was found [14]. Despite the lack of difference in clinical trials, Phase IV data with the dabigitran $150 \mathrm{mg}$ twice daily utilized for stroke prevention in AF still raise bleeding as a significant concern. On December 7, 2011, the FDA initiated an investigation into serious bleeding events associated with dabigatran [37]. Likewise, the Institute for Safe Medication Practices reported that the FDA received nearly three times as many serious and fatal side effects in 2011 with dabigatran as compared to warfarin (3781 versus 1106.) This report also concluded that dabigatran surpassed all other regularly monitored drugs in categories such as deaths (542), hemorrhage (2,367), acute renal failure (291), and stroke (64) and was suspected in 15 cases of liver failure [38].

4.3. Rivaroxaban. Rivaroxaban, an oral direct Factor Xa inhibitor, has been FDA approved for both nonvalvular atrial fibrillation and VTE prevention and in Canada, Europe, 
and the United States for VTE treatment. Presently, three Phase III clinical trials, EINSTEIN-DVT, EINSTEIN-PE, and EINSTEIN-Extension, have been completed to assess the efficacy of rivaroxaban treatment in VTE $[11,39,40]$.

EINSTEIN-DVT was an open label, randomized, multicenter, noninferiority trial enrolling 3449 patients with symptomatic DVT without PE [11]. Patients were eligible if they were 18 years of age or older, had objectively confirmed proximal deep vein thrombosis (DVT), and were without symptomatic pulmonary embolism. Patients were excluded if they received therapeutic doses of low-molecular weight heparin, fondaparinux, or unfractionated-heparin for more than 48 hours or if they had received more than a single dose of a vitamin $\mathrm{K}$ antagonist before randomization, if they had been treated with thrombectomy, a vena cava filter, or a fibrinolytic agents for the current episode of thrombosis, or if they had a contraindications listed in the labeling of the medications used, another indication for warfarin, creatinine clearance $<30 \mathrm{~mL} / \mathrm{min}$; clinically significant liver disease or an alanine aminotransferase level that was three times the upper limit of normal, active, or high risk for bleeding, bacterial endocarditis, systolic blood pressure greater than $180 \mathrm{~mm} \mathrm{Hg}$, or diastolic blood pressure greater than $110 \mathrm{~mm} \mathrm{Hg}$; child bearing potential without proper contraception, pregnancy, or breast-feeding; concomitant use of strong cytochrome P450 3A4 inhibitors; participation in another study within 3 days before screening; and life expectancy of less than 3 months.

In EINSTEIN-DVT, patients were either randomized to either rivaroxaban ( $15 \mathrm{mg}$ orally twice daily for 3 weeks, followed by $20 \mathrm{mg}$ daily for 3 to 12 months) or a parenteral anticoagulant (enoxaparin $1 \mathrm{mg} / \mathrm{kg}$ of body weight subcutaneously twice daily) overlapping with a vitamin $\mathrm{K}$ antagonist such as warfarin or acenocoumarol (dose-adjusted to a target INR of 2.0-3.0) [11]. Parenteral anticoagulation was continued for at least five days until an INR above 2.0 was achieved for two consecutive days. Study endpoints are presented in Table 4. In EINSTEIN DVT, rivaroxaban was noninferior $(P<0.001)$ but not superior $(P=0.08)$ to dose-adjusted warfarin/acenocoumarol managed to a time in TTR of $57.7 \%$ for the primary endpoint of symptomatic recurrent VTE, defined as the composite of DVT or nonfatal or fatal PE. Efficacy was maintained throughout the study, even during the time of transition from twice daily dosing to once daily dosing for rivaroxaban and across all prespecified subgroups. Additionally, the net clinical benefit, taking into account the primary efficacy and safety outcomes, statistically favored rivaroxaban over parenteral anticoagulants plus VKA $(2.9 \%$ versus $4.2 \%$, resp.; $P=0.03$ ). These endpoints were achieved with no additional risk of major bleeding or the primary composite safety endpoint of major bleeding or clinically relevant nonmajor bleeding (Table 5).

EINSTEIN-PE was an open label, single-blinded (outcomes assessment only), randomized, multicenter, noninferiority trial enrolling 4833 patients with symptomatic PE with or without DVT [39]. Inclusion and exclusion criteria were similar to EINSTEIN DVT as were the treatment regimens. The intended duration of treatment was determined by the treating physician before randomization but was either 3 months, 6 months, or 12 months. Study endpoints are presented in Table 4. In EINSTEIN-PE, after a mean study treatment duration of approximately 7.2 months in both groups, rivaroxaban was noninferior $(P<0.003)$, but not superior $(P=0.57)$ to dose-adjusted warfarin/acenocoumarol managed to a time in TTR of $62.7 \%$ for the primary endpoint of symptomatic recurrent VTE, defined as the composite of DVT or nonfatal or fatal PE. Efficacy was maintained throughout the study, even during the time of transition from twice daily dosing to once daily dosing for rivaroxaban and across all prespecified subgroups such as age, sex, presence of absence of obesity, level of renal function, or extent of pulmonary embolism. However, unlike EINSTEIN-DVT, there was no statistical difference in the net clinical benefit taking into account the primary efficacy and safety outcomes between the two groups (Table 4) [11]. These endpoints were achieved with no statistically significant increase in the risk of the primary safety outcome defined as the combination of the first major or clinically relevant nonmajor bleeding episodes but with a statistically significant decrease in the risk of major bleeding between rivaroxaban versus parenteral anticoagulant plus VKA $(1.1 \%$ versus $2.2 \% \mathrm{HR}=0.85(\mathrm{CI}$ : $0.31-0.79) ; P=0.003)$.

EINSTEIN-Extension study was a placebo-controlled, double-blinded, superiority trial designed to determine if extended therapy would decrease the risk of recurrent VTE in patients with confirmed DVT or PE previously treated for 6-12 months with either VKA or rivaroxaban [40]. A total of 1196 patients were enrolled into the trial with $34.1 \%$ of the patients coming from the EINSTEIN DVT study and $19.1 \%$ completing the EINSTEIN PE study while the remaining $47.5 \%$ were referred from outside these studies. Patients were randomized to receive either rivaroxaban $20 \mathrm{mg}$ orally daily or placebo for a duration of 6-12 months. Baseline characteristics were similar between groups. Not surprisingly, rivaroxaban was shown to be superior to placebo in the primary outcome of the prevention of recurrent VTE with a relative risk reduction of $82 \%$ ( $1.3 \%$ versus $7.1 \%$, resp., $\mathrm{HR}=0.18$ (95\% CI 0.09-0.39) $P<0.001)$. The endpoint was achieved without increasing the risk of the primary safety outcome defined as major bleeding $(0.7 \%$ versus $0 \%$; $\mathrm{HR}=$ not reported; $P=0.11)$. However the incidence of the composite endpoint of major bleed or clinically relevant nonmajor bleeding occurred statistically more often in the rivaroxaban group versus placebo (6\% versus $1.2 \%$; $\mathrm{HR}=$ 5.19 (95\% CI 2.3-11.7); $P<0.001$ ). Additionally, the net clinical benefit, taking into account the primary efficacy and safety outcomes, statistically favored rivaroxaban over placebo (2\% versus $7.1 \%$; HR $=0.28$ (95\% CI $0.15-0.53$; $P<0.001)$. Overall, extended therapy prevented 34 recurrent events at the cost of 4 major bleeding events, nonfatal and an increased risk of clinically relevant nonmajor bleeding events compared to placebo.

The EINSTEIN studies, although large and well executed, have the limitation of an open label design lending toward potential bias, although the investigators have indicated that this was unlikely to bias in favor of rivaroxaban. Additionally the use of a placebo control for the extension trial provides little assistance in comparing it against existing therapy, 
TABLE 5: Outcomes of rivaroxaban in VTE Studies $[11,40]$.

\begin{tabular}{|c|c|c|}
\hline Outcome & $\begin{array}{l}\text { EINSTEIN-DVT } \\
\text { (rivaroxaban versus VKA + parenteral ) }\end{array}$ & $\begin{array}{l}\text { EINSTEIN-PE } \\
\text { (rivaroxaban versus VKA + parenteral } \\
\text { agent) }\end{array}$ \\
\hline \multicolumn{3}{|l|}{$\begin{array}{l}\text { Primary efficacy endpoint: } \\
\text { noninferiority }\end{array}$} \\
\hline \multirow{3}{*}{ Recurrent symptomatic VTE } & $2.1 \%$ versus $3.0 \%$, & $2.1 \%$ versus $1.8 \%$ \\
\hline & $\mathrm{HR}=0.68(95 \%$ CI $0.44-1.04)$ & $\mathrm{HR}=1.12(95 \%$ CI $0.75-1.68)$ \\
\hline & $P<0.001$ & $P=0.003$ \\
\hline $\begin{array}{l}\text { \# recurrent events that were } \\
\text { any type of PE }\end{array}$ & 25 versus 24 & 32 versus 27 \\
\hline \multicolumn{3}{|l|}{ Secondary efficacy endpoint: } \\
\hline \multirow{3}{*}{ All-cause mortality } & $2.2 \%$ versus $2.9 \%$ & $2.4 \%$ versus $2.1 \%$ \\
\hline & $\mathrm{HR}=0.67(95 \% \mathrm{CI} 0.44-1.02)$ & $\mathrm{HR}=1.13(95 \%$ CI $0.77-1.65)$ \\
\hline & $P=0.06$ & $P=0.53$ \\
\hline \multirow{3}{*}{ Net clinical benefit } & $2.9 \%$ versus $4.2 \%$ & $3.4 \%$ versus $4.0 \%$ \\
\hline & $\mathrm{HR}=0.67(95 \%$ CI $0.47-0.95)$ & $\mathrm{HR}=0.85(95 \%$ CI $0.63-1.14)$ \\
\hline & $P=0.03$ & $P=0.28$ \\
\hline \multicolumn{3}{|l|}{ Primary safety outcome: } \\
\hline \multirow{3}{*}{$\begin{array}{l}\text { Major bleeding or clinically relevant } \\
\text { nonmajor bleeding }\end{array}$} & $8.1 \%$ versus $8.1 \%$ & $10.3 \%$ versus $11.4 \%$ \\
\hline & $\mathrm{HR}=0.97(99 \% \mathrm{CI} 0.76-1.22)$ & $\mathrm{HR}=0.90(95 \% \mathrm{CI} 0.76-1.07)$ \\
\hline & $P=0.77$ & $P=0.23$ \\
\hline \multirow{3}{*}{ Major bleeding ${ }^{\wedge}$} & $0.8 \%$ versus $1.2 \%$ & $1.1 \%$ versus $2.2 \%$ \\
\hline & $\mathrm{HR}=0.65(95 \% \mathrm{CI} 0.33-1.30)$ & $\mathrm{HR}=0.49(95 \%$ CI $0.31-0.79)$ \\
\hline & $P=0.21$ & $P=0.003$ \\
\hline \multirow{2}{*}{$\begin{array}{l}\text { Clinically relevant nonmajor } \\
\text { bleeding }^{\wedge \wedge}\end{array}$} & $7.36 \%$ versus $7.0 \%$ & $9.5 \%$ versus $9.8 \%$ \\
\hline & (statistics NR) & (statistics NR) \\
\hline Warfarin TTR & $57.7 \%$ & $62.7 \%$ \\
\hline Study conclusion & $\begin{array}{l}\text { Rivaroxaban is noninferior to warfarin. } \\
\text { Rivaroxaban is not superior to warfarin. }\end{array}$ & $\begin{array}{l}\text { Rivaroxaban is noninferior to warfarin. } \\
\text { Rivaroxaban is not superior to warfarin }\end{array}$ \\
\hline
\end{tabular}

NR: not reported, TTR: time in therapeutic range.

${ }^{\wedge}$ Major bleeding as defined in EINSTEIN: Clinical overt bleeding, with associated fall in hemoglobin of at least $2.0 \mathrm{~g}$ per deciliter, the need for transfusion of 2 or more units of red blood cells, involving a critical site or was fatal.

${ }^{\wedge}$ Clinically relevant nonmajor bleeding as defined in EINSTEIN: overt bleeding that did not meet the criteria for major bleeding but was associated with medical intervention, unscheduled contact with a physician, interruption or discontinuation of a study drug or discomfort, or impairment of activities of daily life.

warfarin, and its relatively short duration of follow-up (mean of 9 months) does not clearly address safety and efficacy for years of chronic therapy. Although secondary analyses of the EINSTEIN studies did not identify any signals or concern for liver toxicity or evidence of unexpected thrombotic events, longer duration of therapy to address a chronic lifelong condition would have been valuable $[11,40]$. The most recent update of the ACCP Guidelines on Antithrombotic Therapy from 2012 [2] suggests extending warfarin therapy beyond 3 months in patients with unprovoked VTE who have low-tomoderate risk of bleeding, and if bleeding risk is high, then therapy is limited to 3 months. Having a trial that compares the bleeding risk of a new agent against standard VKA therapy during extension of therapy would help refine these recommendations even further, especially if bleeding risk is lower with the NOACs compared to VKA. The EINSTEIN studies included a high percentage of unprovoked events (range between $60 \%$ and $70 \%$ ) and $4-6 \%$ of patients with active cancer making this a moderate-to-high risk population for recurrent events that could have effectively addressed many of these questions. Further research is therefore recommended for the chronic use of rivaroxaban for VTE prevention after an initial event.

\section{Conclusions}

Based upon the data presented, dabigatran and apixaban cannot be recommended until further safety and efficacy data are available. Rivaroxaban however, represents the most promising of the NOACs for treatment of $\mathrm{PE}$ and is indicted in Canada and Europe for this indication and recently received FDA approval for this indication [21]. Although the ACCP 2012 Guidelines on Antithrombotic Therapy published in February 2012 indicated warfarin as the agent with the 
most sufficient data, the results of the EINSTEIN PE were not yet available at the time of publication [2]. With the approval of rivaroxaban for treatment of VTE (DVT and PE) now in the USA, Canada, and Europe, this offers yet another treatment option. However, selection of rivaroxaban over warfarin must take into account a full assessment of an individual's renal function, concomitant drug therapy, and risk for bleeding. Although rivaroxaban was shown to be noninferior to warfarin in both the DVT and PE studies $[11,40]$, the risk of major bleeding was statistically lower only in the PE study [40]. The issue of bleeding risk is paramount in the decision of appropriate patient selection due to the lack of reversal agent. Comparative efficacy is also warranted in the setting of specialized anticoagulation clinics where the TTR is generally above 70 percent [41-45] as the current studies achieved only a 57-62\% TTR. Lastly, long-term (>12 months) comparison data against standard warfarin therapy are lacking, and therefore recommendations for extended therapy beyond the acute phase of treatment cannot be made.

Based on the strength of available evidence, warfarin proves to be a monitorable, reversible, and effective agent in patients across the spectrum of concurrent disease and should remain the first-line option in conjunction with appropriate parenteral anticoagulation for the treatment of pulmonary embolism, especially in patients who require chronic therapy or therapy beyond 3 months. However, rivaroxaban represents a viable alternative in patients with less than optimal INR control (TTR $<60 \%$ ) or in whom warfarin monitoring and management is not possible.

\section{Conflict of Interests}

The authors report that they have no conflict of interests.

\section{Acknowledgment}

The authors would like to acknowledge Dr. Kimberly Zammit for her editorial assistance.

\section{References}

[1] D. Hawkins, "Pharmacoeconomics of thrombosis management," Pharmacotherapy, vol. 24, no. 7, part 2, pp. 95S-99S, 2004.

[2] S. M. Bates, R. Jaeschke, S. M. Stevens et al. et al., "Diagnosis of DVT: antithrombotic therapy and prevention of thrombosis, 9th ed: American College of Chest physicians evidence-based clinical practice guidelines," Chest, vol. 141, supplement 2, pp. e351S-e418S, 2012.

[3] J. Edelsberg, D. Ollendorf, and G. Oster, "Venous thromboembolism following major orthopedic surgery: review of epidemiology and economics," American Journal of Health-System Pharmacy, vol. 58, no. 2, pp. S4-S13, 2001.

[4] E. Nutescu, "Characteristics of novel anticoagulants and potential economic implications," American Journal of Managed Care, vol. 17, pp. S27-S32, 2011.

[5] C. Kearon, "Natural history of venous thromboembolism," Circulation, vol. 107, no. 23, supplement 1, pp. I22-I30, 2003.
[6] N. Oake, D. A. Fergusson, A. J. Forster, and C. Van Walraven, "Frequency of adverse events in patients with poor anticoagulation: a meta-analysis," Canadian Medical Association Journal, vol. 176, no. 11, pp. 1589-1594, 2007.

[7] A. J. Rose, A. Ozonoff, R. W. Grant, L. E. Henault, and E. M. Hylek, "Epidemiology of subtherapeutic anticoagulation in the United States," Circulation: Cardiovascular Quality and Outcomes, vol. 2, no. 6, pp. 591-597, 2009.

[8] L. Hamby, W. B. Weeks, and C. Malikowski, "Complications of warfarin therapy: causes, costs, and the role of the anticoagulation clinic," Effective Clinical Practice, vol. 3, no. 4, pp. 179-184, 2000.

[9] A. M. Holbrook, J. A. Pereira, R. Labiris et al., "Systematic overview of warfarin and its drug and food interactions," Archives of Internal Medicine, vol. 165, no. 10, pp. 1095-1106, 2005.

[10] S. Murin, P. S. Romano, and R. H. White, "Comparison of outcomes after hospitalization for deep venous thrombosis or pulmonary embolism," Thrombosis and Haemostasis, vol. 88, no. 3, pp. 407-414, 2002.

[11] R. Bauersachs, S. D. Berkowitz, B. Brenner et al. et al., "Oral rivaroxaban for symptomatic venous thromboembolism," The New England Journal of Medicine, vol. 363, no. 26, pp. 24992510, 2010.

[12] H. R. Buller, A. W. A. Lensing, M. H. Prins et al., "A doseranging study evaluating once-daily oral administration of the factor Xa inhibitor rivaroxaban in the treatment of patients with acute symptomatic deep vein thrombosis: the Einstein DVT dose-ranging study," Blood, vol. 112, no. 6, pp. 2242-2247, 2008.

[13] "Secondary Prevention of Venous Thrombo Embolism (VTE)," NCT00329238, 2012, http://www.clinicaltrials.gov/ct2/show/ NCT00329238? term=NCT00329238\&rank=1.

[14] S. Schulman, C. Kearon, A. K. Kakkar et al., "Dabigatran versus warfarin in the treatment of acute venous thromboembolism," The New England Journal of Medicine, vol. 361, no. 24, pp. 23422352, 2009.

[15] Elise S. Eerenberg, Pieter W. Kamphuisen, Meertien K. Sijpkens, Joost C. Meijers, Harry R. Buller, and M. Levi, "Reversal of rivaroxaban and dabigatran by prothrombin complex concentrate: a randomized, placebo-controlled, crossover study in healthy subjects," Circulation, vol. 124, no. 14, pp. 1573-1579, 2011.

[16] J. van Ryn, J. van Ryn, M. Kink-Eiband, and A. Clemens, "The successful reversal of dabigatran-induced bleeding by coagulation factor concentrates in a rat tail bleeding model do not correlate with ex vivo markers of anticoagulation," in Proceedings of the 53rd American Society of Hematology Annual Meeting, San Diego, Calif, USA, December 2011, Poster abstract 2216.

[17] G. William and R. Aaron, "Reversing dabigatran with feiba in a patient with a transseptal perforation during cardiac ablation," Critical Care Medicine, vol. 39, no. 12, p. 243, 2011.

[18] Pradaxa (Dabigatran) Package Insert, Boehringer Ingelheim Pharmaceuticals, Ridgefield, Conn, USA, 2012.

[19] S. Schulman and M. A. Crowther, "How I treat with anticoagulants in 2012: new and old anticoagulants, and when and how to switch," Blood, vol. 119, no. 13, pp. 3016-3023, 2012.

[20] M. Levi, J. H. Levy, H. F. Andersen, and D. Truloff, "Safety of recombinant activated factor VII in randomized clinical trials," New England Journal of Medicine, vol. 363, no. 19, pp. 1791-1800, 2010. 
[21] Xarelto (Rivaroxaban) Package Insert, Janssen Pharmaceuticals, Titusville, NJ, USA, 2011.

[22] J. van Ryn, D. Ruehl, H. Priepke, N. Hauel, and W. Wienen, "Reversibility of the anticoagulant effect of high doses of dabigatran by rFVIIa or PCC," in Proceedings of the 13th Congress of the European Hematology Association, June 2008.

[23] N. Raghavan, C. E. Frost, Z. Yu et al., "Apixaban metabolism and pharmacokinetics after oral administration to humans," Drug Metabolism and Disposition, vol. 37, no. 1, pp. 74-81, 2009.

[24] B. I. Eriksson, D. J. Quinlan, and J. I. Weitz, "Comparative pharmacodynamics and pharmacokinetics of oral direct thrombin and factor Xa inhibitors in development," Clinical Pharmacokinetics, vol. 48, no. 1, pp. 1-22, 2009.

[25] J. Stangier, "Clinical pharmacokinetics and pharmacodynamics of the oral direct thrombin inhibitor dabigatran etexilate," Clinical Pharmacokinetics, vol. 47, no. 5, pp. 285-295, 2008.

[26] Coumadin (Wafarin) Package Insert, Bristol Meyers Squibb Company, Princeton, NJ, USA, 2010.

[27] R. Marlu, E. Hodaj, A. Paris, P. Albaladejo, J. L. Crackowski, and G. Pernod, "Effect of non-specific reversal agents on anticoagulant activity of dabigatran and rivaroxaban: a randomised crossover ex vivo study in healthy volunteers," Thrombosis and Haemostasis, vol. 108, no. 2, pp. 217-224, 2012.

[28] W. Zhou, S. Schwarting, S. Illanes et al., "Hemostatic therapy in experimental intracerebral hemorrhage associated with the direct thrombin inhibitor dabigatran," Stroke, vol. 42, no. 12, pp. 3594-3599, 2011.

[29] T. E. Warkentin, P. Margetts, S. J. Connolly, A. Lamy, C. Ricci, and J. W. Eikelboom, "Recombinant factor VIIa (rFVIIa) and hemodialysis to manage massive dabigatran-associated postcardiac surgery bleeding," Blood, vol. 119, no. 9, pp. 21722174, 2012.

[30] H. J. Kolde, "Determining the exact concentration of direct thrombin inhibitors (DTI) in plasma," 2012, http://www.clionline.com/featured-articles/determining-the-exact-concentration-of-direct-thrombin-inhibitors-dti-in-plasma/index.html.

[31] Eliquis (Apixaban) Prescribing Information, Bristol Meyers Squibb Company, Princeton, NJ, USA, 2012.

[32] L. Wang, D. Zhang, N. Raghavan et al., "In vitro assessment of metabolic drug-drug interaction potential of apixaban through cytochrome 450 phenotyping, inhibition, and induction studies," Drug Metabolism and Disposition, vol. 38, no. 3, pp. 448458, 2010.

[33] "Efficacy and Safety Study of Apixaban for the Treatment of Deep Vein Thrombosis or Pulmonary Embolism," 2012, http://www.clinicaltrials.gov/ct2/show/NCT00643201?term= NCT00643201\&rank=1.

[34] G. Agnelli, H. R. Buller, A. Cohen et al., "Apixaban for extended treatment of venous thromboembolism," The New England Journal of Medicine, 2012.

[35] S. Schulman, A. K. Kakkar, S. M. Schellong et al., "A Randomized trial of dabigatran versus warfarin in the treatment of acute venous thromboembolism (RE-COVER II)," in Proceedings of the 53rd ASH Annual Meeting and Exposition, San Diego, Calif, USA, December 2011.

[36] "Twice-daily Oral Direct Thrombin Inhibitor Dabigatran Etexilate in the Long Term Prevention of Recurrent Symptomatic VTE," 2012, http://www.clinicaltrials.gov/ct2/show/ NCT00558259? term=NCT00558259\&rank=1.

[37] "Pradaxa (dabigatran etexilate mesylate): Drug Safety Communication-Safety Review of Post-Market Reports of Serious
Bleeding Events," 2012, http://www.fda.gov/Safety/MedWatch/ SafetyInformation/SafetyAlertsforHumanMedicalProducts/ ucm 282820.htm.

[38] “Quarter Watch 2011 Annual Report," Instiute for Safe Medication Practices.

[39] H. R. Buller, M. H. Prins, A. W. Lensin et al. et al., "Oral rivaroxaban for the treatment of symptomatic pulmonary embolism," The New England Journal of Medicine, vol. 366, no. 14, pp. 12871297, 2012.

[40] E. Romualdi, M. P. Donadini, and W. Ageno, "Oral rivaroxaban after symptomatic venous thromboembolism: the continued treatment study (EINSTEIN-Extension study)," Expert Review of Cardiovascular Therapy, vol. 9, no. 7, pp. 841-844, 2011.

[41] K. M. Rudd and J. G. Dier, "Comparison of two different models of anticoagulation management services with usual medical care," Pharmacotherapy, vol. 30, no. 4, pp. 330-338, 2010.

[42] S. M. Garabedian-Ruffalo, D. R. Gray, M. J. Sax, and R. L. Ruffalo, "Retrospective evaluation of a pharmacist-managed warfarin anticoagulation clinic," American Journal of Hospital Pharmacy, vol. 42, no. 2, pp. 304-308, 1985.

[43] S. J. A. Wilson, P. S. Wells, M. J. Kovacs et al., "Comparing the quality of oral anticoagulant management by anticoagulation clinics and by family physicians: a randomized controlled trial," Canadian Medical Association Journal, vol. 169, no. 4, pp. 293298, 2003.

[44] J. E. Lafata, S. A. Martin, S. Kaatz, and R. E. Ward, "Anticoagulation clinics and patient self-testing for patients on chronic warfarin therapy: a cost-effectiveness analysis," Journal of Thrombosis and Thrombolysis, vol. 9, supplement 1, pp. S13S19, 2000.

[45] A. G. Staresinic, C. A. Sorkness, B. M. Goodman, and D. W. Pigarelli, "Comparison of outcomes using 2 delivery models of anticoagulation care," Archives of Internal Medicine, vol. 166, no. 9, pp. 997-1006, 2006. 


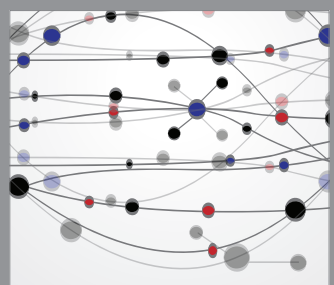

The Scientific World Journal
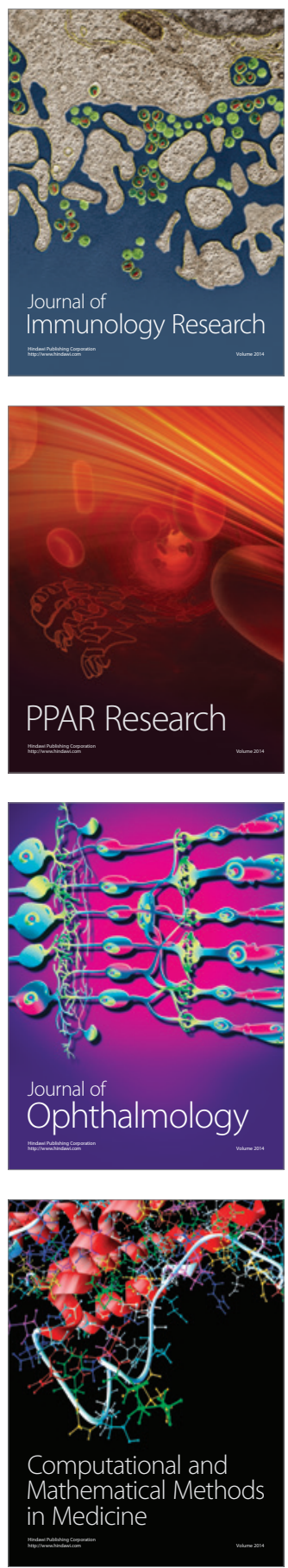

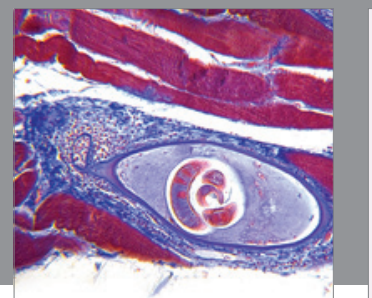

Gastroenterology

Research and Practice
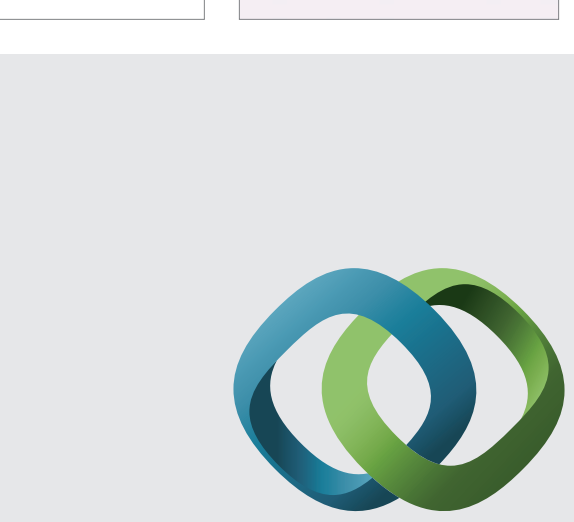

\section{Hindawi}

Submit your manuscripts at

http://www.hindawi.com
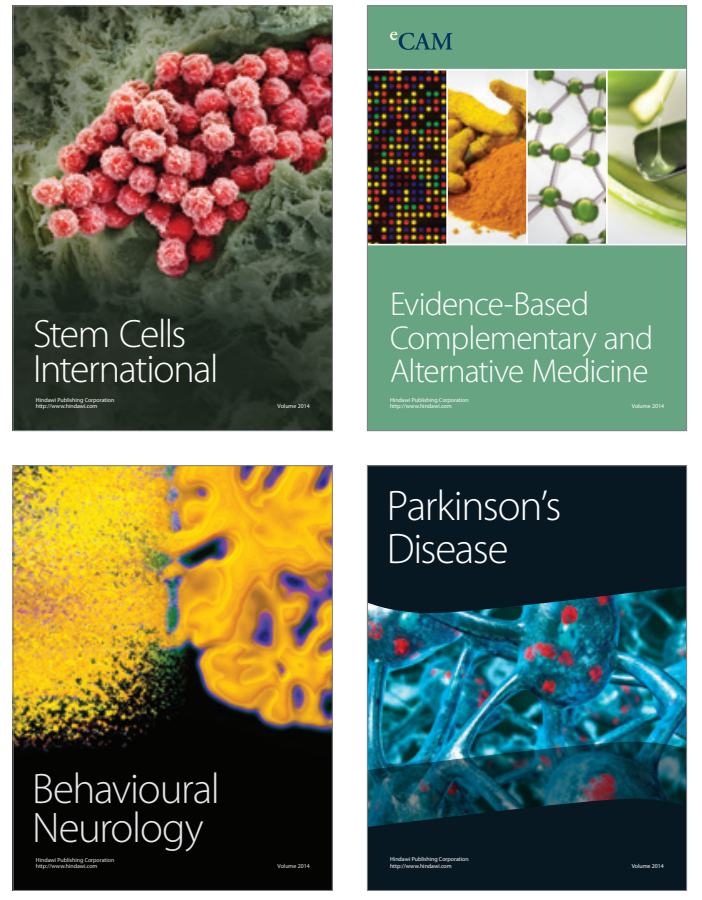
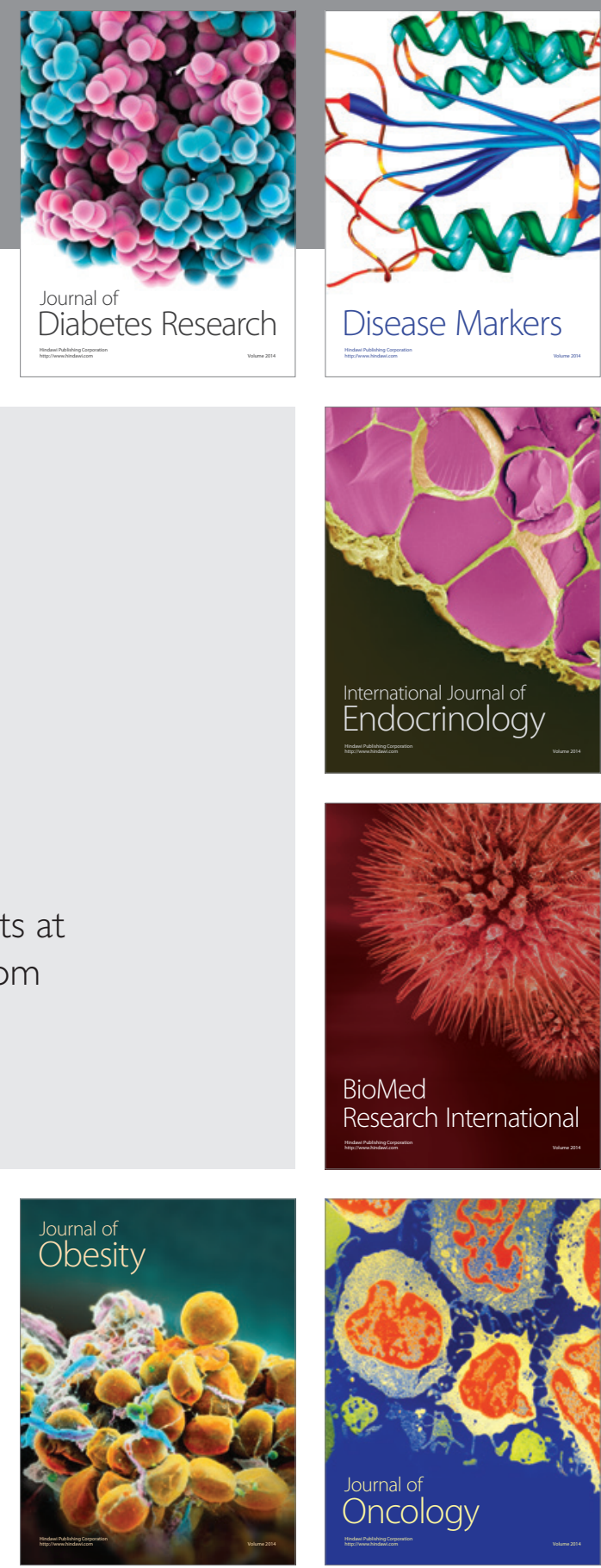

Disease Markers
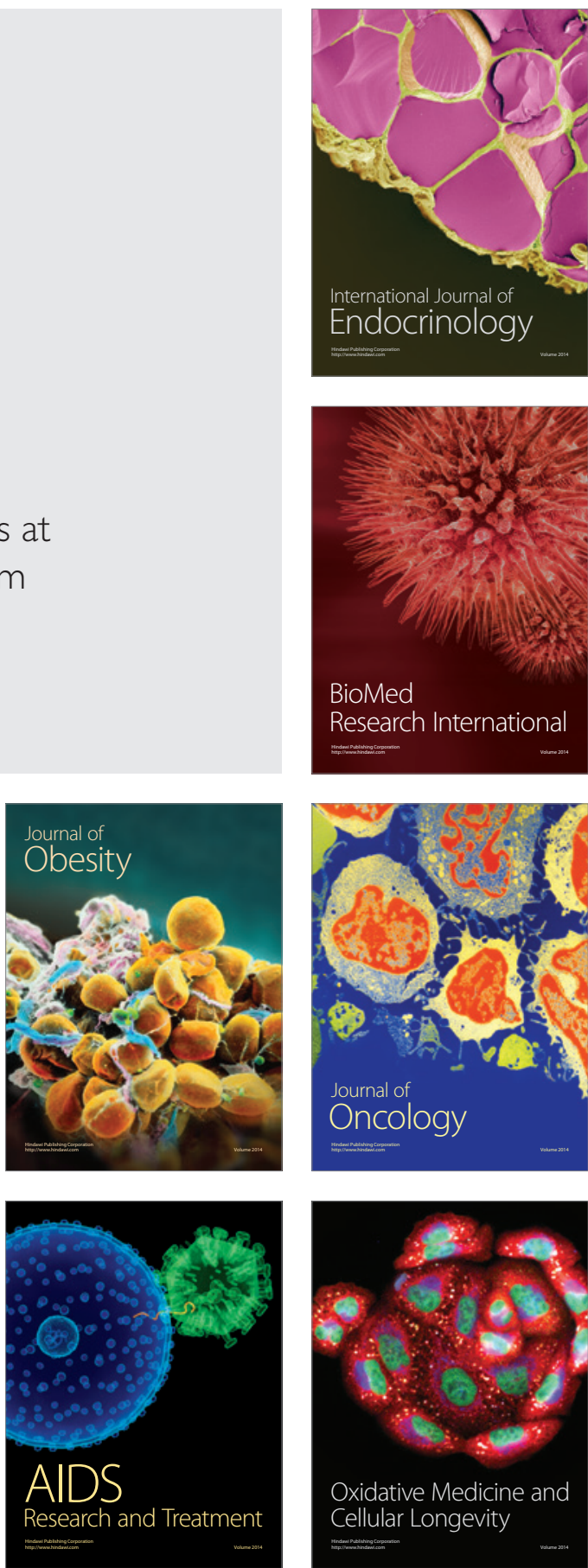